\title{
Transient HDO rovibrational satellite peaks in solid parahydrogen: evidence of hydrogen atoms or vacancies?
}

\author{
William R. Wonderly and David T. Anderson \\ Department of Chemistry, University of Wyoming, Laramie, WY 82071-3838, USA \\ E-mail: danderso@uwyo.edu
}

Received March 18, 2012

\begin{abstract}
We present FTIR studies of the $193 \mathrm{~nm}$ photolysis of fully deuterated formic acid (DCOOD) isolated in solid parahydrogen at $1.9 \mathrm{~K}$ which show evidence of transient HDO rovibrational satellite peaks. The S1 and S2 satellite peaks are readily detected for $a$-type $\left(1_{01} \leftarrow 0_{00}\right)$ rovibrational transitions of HDO either during or immediately after photolysis. Intensity measurements show the HDO $b$-type $\left(1_{11} \leftarrow 0_{00}\right)$ rovibrational transitions have satellite peaks as well, but due to the greater linewidth of these absorptions, the satellite peaks cannot be spectroscopically resolved from the monomer transition and are therefore difficult to detect. These newly identified HDO satellite peaks may result from the HDO photoproduct being formed next to an $\mathrm{H}$ atom or a vacancy in the parahydrogen solid. The development of the infrared spectroscopy of these satellite peaks can provide a new means to study radiation effects on low-temperature hydrogen solids doped with chemical species.
\end{abstract}

PACS: 61.80.-X Physical radiation effects, radiation damage;

67.80.ff Molecular hydrogen and isotopes;

67.80.dj Defects, impurities, and diffusion.

Keywords: solid hydrogen, impurities, diffusion.

\section{Introduction}

In preliminary studies of the $193 \mathrm{~nm}$ in situ photolysis of different isotopomers of formic acid $(\mathrm{HCOOH}$ and DCOOD) in solid parahydrogen $\left(p \mathrm{H}_{2}\right)$ at $1.9 \mathrm{~K}$, we identified satellite peaks in close proximity to the $a$-type rovibrational peaks of the $\mathrm{H}_{2} \mathrm{O}$ and HDO photoproducts [1]. Specifically, we observe the $\mathrm{S} 1$ satellite peak approximately $1 \mathrm{~cm}^{-1}$ lower and the $\mathrm{S} 2$ peak around $2 \mathrm{~cm}^{-1}$ higher in energy than the monomer $a$-type $\mathrm{R}(0)$ rovibrational peak for $\mathrm{HDO}$ and $\mathrm{H}_{2} \mathrm{O}$, but not $\mathrm{D}_{2} \mathrm{O}$. Even when the sample is maintained at $1.9 \mathrm{~K}$ after photolysis the intensity of these satellite peaks decay slowly with a time constant of 121(7) $\mathrm{min}$ [1]. Photoexcitation of $\mathrm{HCOOH}$ in the gas phase at $193 \mathrm{~nm}$ leads to direct dissociation to give $\mathrm{HCO}+\mathrm{OH}$ as the dominant photochannel $[2,3]$. In the preliminary studies we used the lack of satellite peaks for $\mathrm{D}_{2} \mathrm{O}$ to assign the observed satellite peaks to $\mathrm{H} \cdots \mathrm{HDO}$ or $\mathrm{H}^{\cdots} \cdot \mathrm{H}_{2} \mathrm{O}$ radical clusters that form as by-products of the $193 \mathrm{~nm}$ photochemistry via reactions of the $\mathrm{OD}$ or $\mathrm{OH}$ photofragments with the $p \mathrm{H}_{2}$ host [1]. However, at that time we were unable to definitively explain why we did not observe the analogous satellite peaks associated with the $b$-type $\mathrm{R}(0)$ rovibrational transitions of either $\mathrm{HDO}$ or $\mathrm{H}_{2} \mathrm{O}$. Further, the spectroscopic data is also consistent with the
HDO or $\mathrm{H}_{2} \mathrm{O}$ photoproducts being produced next to a vacancy left by the reacting $p \mathrm{H}_{2}$ molecule. Therefore, in this work we more fully develop our understanding of the infrared (IR) spectroscopy of the HDO satellite peaks.

The rovibrational bands of asymmetric top molecules such as $\mathrm{H}_{2} \mathrm{O}$ or HDO are classified by whether the transition dipole moment responsible for the absorption has a projection along the $A, B$, or $C$ inertial axes of the molecule [4]. For $\mathrm{H}_{2} \mathrm{O}$ the symmetry axis corresponds to the $B$ axis and the $A$ axis lies in the plane of the molecule, so the $v_{2}$ bend fundamental is a $b$-type band and the $v_{3}$ asymmetric stretch is an $a$-type band [5]. For HDO where the symmetry is lowered by isotopic substitution, the $v_{2}$ bend is now a mixed $a / b$-type band since the transition dipole has projections along both inertial axes. Furthermore, HDO and $\mathrm{H}_{2} \mathrm{O}$ freely rotate in solid $p \mathrm{H}_{2}$ such that at the low temperatures where solid $p \mathrm{H}_{2}$ is stable, the overwhelming majority of $\mathrm{HDO}$ or $\mathrm{H}_{2} \mathrm{O}$ molecules populate only the lowest rotational level, namely $J_{K_{a} K_{c}}=0_{00}$. Under these conditions, the rovibrational bands collapse into a single rovibrational transition which is $b$-type $\left(1_{11} \leftarrow 0_{00}\right)$ for the $v_{2}$ bend and $a$-type $\left(1_{01} \leftarrow 0_{00}\right)$ for the $v_{3}$ asymmetric stretch of $\mathrm{H}_{2} \mathrm{O}$, but both $b$ - and $a$-type rovibrational transitions are observed for HDO for the $v_{2}$ and $v_{3}$ vibrational fundamen- 
tals [5]. In this paper we show that satellite peaks are also present for the $b$-type rovibrational transitions of HDO, but due to the greater full width half maximum of the $b$-type transitions of HDO in solid $p \mathrm{H}_{2}$, the satellite peaks cannot be resolved from the monomer transition and thus are much more difficult to detect using FTIR.

The assignment of the carriers of these HDO satellite peaks is important if we are to develop new methods to study the in situ photochemistry of chemically doped $\mathrm{pH}_{2}$ quantum solids. If the HDO satellite peaks presented in this work are due to $\mathrm{H} \cdots \mathrm{HDO}$ radical clusters then these satellite peaks could be used to study the mechanism by which in situ photochemical reactions proceed and also provide a new spectroscopic handle on $\mathrm{H}$ atoms in solid $p \mathrm{H}_{2}$. Indeed, $\mathrm{H}$ atoms were first detected and shown to be mobile in solid hydrogen samples at liquid helium temperatures nearly 30 years ago [6-8]. Much of the subsequent progress in our understanding of $\mathrm{H}$ atoms trapped in solid $p \mathrm{H}_{2}$ has been provided by the electron spin resonance (ESR) studies of Kumada and co-workers [9-14]. These studies led first to the determination that the $\mathrm{H}$ atoms diffuse via a chemical diffusion mechanism by which the $\mathrm{H}$ atom moves by sequential $\mathrm{H}+\mathrm{H}_{2} \rightarrow \mathrm{H}_{2}+\mathrm{H}$ chemical exchange reactions [13]. Through a variety of ESR measurements, both the rates of $\mathrm{H}$ atom diffusion and $\mathrm{H}+\mathrm{H} \rightarrow \mathrm{H}_{2}$ recombination can be studied separately [12]. These measurements show that for highly enriched $\mathrm{pH}_{2}$ samples (i.e., low orthohydrogen $\left(\mathrm{oH}_{2}\right)$ concentrations) and at low temperature, the recombination rate is too slow to be explained by the diffusion rate which suggests that the $\mathrm{H}$ atoms do not recombine efficiently under these conditions [12]. Fushitani and Momose used FTIR spectroscopy [15] to measure the $\mathrm{H}$ atom diffusion rate by studying the $\mathrm{H}+\mathrm{NO} \rightarrow \mathrm{HNO}$ tunneling reaction after the in situ photolysis of NO in solid $p \mathrm{H}_{2}$ at $5.2 \mathrm{~K}$. In these studies the $\mathrm{H}$ atoms are produced as by-products of the NO photolysis, and the H-atom diffusion rate is determined from the growth in the IR absorption intensity of HNO. Andrews and co-workers studied the induced IR transitions in solid hydrogen (and enriched $p \mathrm{H}_{2}$ solids) produced by the presence of an $\mathrm{H}$ atom either by laser ablating metal atoms into the solids or by direct condensation of hydrogen gas subjected to tesla coil discharge in a quartz tube $[16,17]$. In these studies $H$ atom induced peaks in $\mathrm{pH}_{2}$ were identified at $4151.8 \mathrm{~cm}^{-1}$ which show intensity half-lives compatible with the ESR measurements.

The other possibility that we consider is the HDO satellite peaks are caused by HDO produced next to a vacancy. As discussed in our earlier work, we only observe HDO satellite peaks for irradiated samples suspected to produce OD photofragments that can react with the $\mathrm{pH}_{2}$ host to form HDO. In this case the products of the $\mathrm{OD}+p \mathrm{H}_{2} \rightarrow$ $\rightarrow \mathrm{HDO}+\mathrm{H}$ chemical reaction get trapped next to each other in the solid, and may lead to the formation of $\mathrm{H} \cdots \mathrm{HDO}$ radical clusters. However, if the $\mathrm{H}$ atom escapes somehow, then the HDO molecule might be trapped next to the vacancy left behind by the $\mathrm{pH}_{2}$ molecule that reacted. This would mean that the in situ photolysis produces vacancies and these vacancies may play a role in subsequent $\mathrm{H}$ atom reactions that occur after photolysis. Indeed vacancies are central to the original proposal by Andreev and Lifshitz that under certain conditions quantum crystals may show a new super state of matter, a socalled supersolid [18]. A number of studies have explored defect ( $\mathrm{H}$ atom, $\mathrm{oH}_{2}, \mathrm{H}_{2}^{-}$) diffusion in hydrogen crystals, but none have been able to directly quantify the number of vacancies $[19,20]$. If the HDO satellite peaks reflect HDO next to vacancies, then in situ photolysis of certain precursor molecules can lead to the generation of nonequilibrium vacancy populations at low temperature which may influence the diffusion of chemical impurities after photolysis.

\section{Experimental details}

Our experimental apparatus is described in detail elsewhere $[1,21]$; briefly, we grow millimeters thick, chemically doped $p \mathrm{H}_{2}$ crystals via co-deposition of independent gas streams of dopant (e.g., DCOOD) and $p \mathrm{H}_{2}$ host onto a $\mathrm{BaF}_{2}$ optical substrate maintained at approximately $2.5 \mathrm{~K}$ inside a sample-in-a-vacuum liquid helium bath cryostat. The host in these studies is enriched to approximately 99.97\% pure $\mathrm{pH}_{2}$ levels using a variable temperature ortho/para catalytic converter operated near $14.0 \mathrm{~K}$ during deposition. FTIR spectroscopy at $0.05 \mathrm{~cm}^{-1}$ resolution is performed on the sample using a normal incidence transmission optical setup. The measured integrated intensities of specific solid $\mathrm{pH}_{2}$ IR absorptions allow us to determine the IR path length through the sample [22], which permits the concentration of species within the crystal to be determined using Beer's law. Photolysis is achieved using the $193 \mathrm{~nm}$ output of an ArF excimer laser (Gam Laser EX5) configured to pass through the sample at an angle of $45^{\circ}$ with respect to the FTIR beam. This optical setup permits FTIR spectra to be recorded within the photolyzed region of the crystal either during or immediately after $193 \mathrm{~nm}$ irradiation. The DCOOD concentration is the average value determined from the $v_{1}$ (OD stretch) and $v_{6}$ (deformation) peaks with integrated absorption intensities of 36.9 and $56.8 \mathrm{~km} / \mathrm{mol}$, respectively [23].

\section{Experimental results and discussion}

Shown in Fig. 1 is IR difference spectra of the $a$-type $\left(1_{01} \leftarrow 0_{00}\right)$ and $b$-type $\left(1_{11} \leftarrow 0_{00}\right)$ rovibrational transitions of the $v_{2}$ mode of HDO for a DCOOD doped $p \mathrm{H}_{2}$ sample that is subjected to $193 \mathrm{~nm}$ photolysis. The difference IR spectra (after minus before) shown as traces (a) through (d) in Fig. 1 are generated using a "before" spectrum recorded just prior to photolysis, and a series of "after" spectra recorded with $3 \mathrm{~min}$ acquisition times 


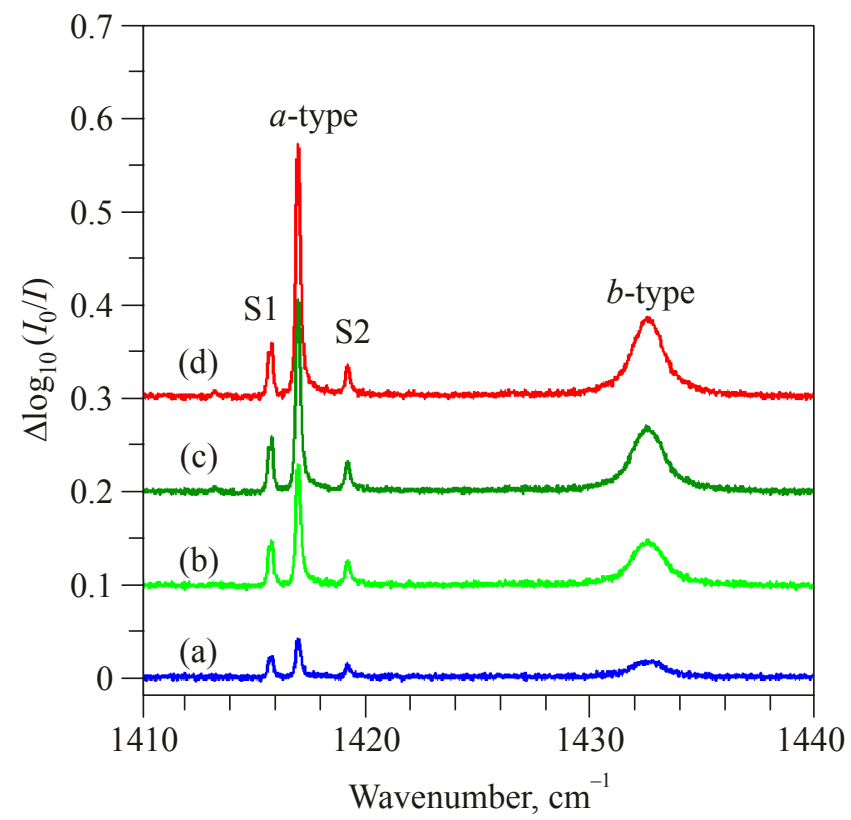

Fig. 1. (Color online) A series of IR difference absorption spectra (after minus before) displaying the growth of the $v_{2}$ HDO $a$-type and $b$-type rovibrational peaks during photolysis of a DCOOD doped $p \mathrm{H}_{2}$ sample. The "before" spectrum is recorded just before photolysis is started, and the "after" spectra are recorded at $1.93 \mathrm{~K}$ during the $14.4 \mathrm{~min}(37 \mathrm{~mW}$ at $250 \mathrm{~Hz}) \mathrm{UV}$ irradiation of the sample. Each FTIR scan has a 3 min acquisition time $\left(0.05 \mathrm{~cm}^{-1}\right.$ and 16 co-added scans) and traces (a) through (d) were recorded at increasing times $(1.5,4.8,8.2,11.5 \mathrm{~min})$ after photolysis is started. Note the growth of the S1 and S2 satellite peaks close to the $a$-type transition. The sample is $0.26(1) \mathrm{cm}$ thick with an initial DCOOD concentration of 30(5) ppm. Spectra are offset vertically for ease of comparison.

$\left(0.05 \mathrm{~cm}^{-1}\right.$ and 16 co-added scans) centered at $1.5,4.8$, 8.2 , and $11.5 \mathrm{~min}$ after the start of the photolysis laser. All these spectra are recorded during the $193 \mathrm{~nm}$ photolysis of a $30 \mathrm{ppm}$ DCOOD doped $\mathrm{pH}_{2}$ solid $\left(99.97 \% \mathrm{pH}_{2}\right)$ at $1.93 \mathrm{~K}$. We estimate that the solid $p \mathrm{H}_{2}$ contains $6.4 \mathrm{ppm}$ of $\mathrm{DCOOH}, 1.3 \mathrm{ppm}$ of HCOOD, and $0.7 \mathrm{ppm}$ of $\mathrm{HCOOH}$ prior to photolysis due to isotopic scrambling of the DCOOD. As can be seen in Fig. 1, both the $a$-type and $b$-type rovibrational transitions for the $v_{2}$ bend of HDO increase in intensity with photolysis. These two rovibrational transitions are labeled $a$-type and $b$-type, respectively, to indicate the orientation of the transition dipole moment with respect to the inertial axis of the HDO molecule and to account for the selection rules for that particular rovibrational transition. However, the S1 and S2 satellite peaks are only clearly detected for the $a$-type rovibrational transition while no satellite peaks are detected for the $b$-type transition. As discussed in the Introduction, this observation confused us in preliminary studies where we speculated the lack of $b$-type satellite peaks was due either to some sort of selection rules or related to the breadth of the two rovibrational absorptions [1]. These two rovibrational transitions for HDO monomers isolated in solid $p \mathrm{H}_{2}$ have been well characterized previously [5,24,25], however the reason that the two peaks have such different full width half maximum (fwhm) values has not been considered in detail $[5,25]$. The greater fwhm of the $b$-type transition $\left(1.8 \mathrm{~cm}^{-1}\right)$ compared to the $a$-type absorption $\left(0.27 \mathrm{~cm}^{-1}\right)$ is likely due to faster rotational relaxation of the $1_{11}$ rotational state compared to the $1_{01}$ state, but a detailed study of these differences is beyond the scope of the present work. Here we only point out that if the satellite peaks for the $b$-type rovibrational transitions come at comparable shifts from the monomer rovibrational transition, then the satellite peaks will be difficult to detect since they will not be cleanly resolved, as is the case for the $a$-type transition.

To clearly show that S1 and S2 satellite peaks are detected for $a$-type rovibrational peaks of all the fundamental modes of HDO, we show the analogous difference spectra for the $v_{1}$ and $v_{3}$ HDO rovibrational peaks in Fig. 2. These two $a$-type transitions show that the shifts of the satellite peaks from the monomer rovibrational transition are quite similar for both rovibrational transitions (and with the $v_{2}$ $a$-type peak in Fig. 1). Specifically, the S1 peak always comes at lower wavenumbers and the S2 at higher wavenumbers compared to the HDO monomer transition. This seems to indicate that the shift of the satellite peaks from the corresponding monomer transition depends on the upper rotational state, which is the same for all these transitions, and not too strongly on the specific vibrational state. The spectra shown in Fig. 2 do not have as high a signalto-noise $(\mathrm{S} / \mathrm{N})$ ratio as the previously published spectra [1], and this is due in part to the greater noise of the MCT de-

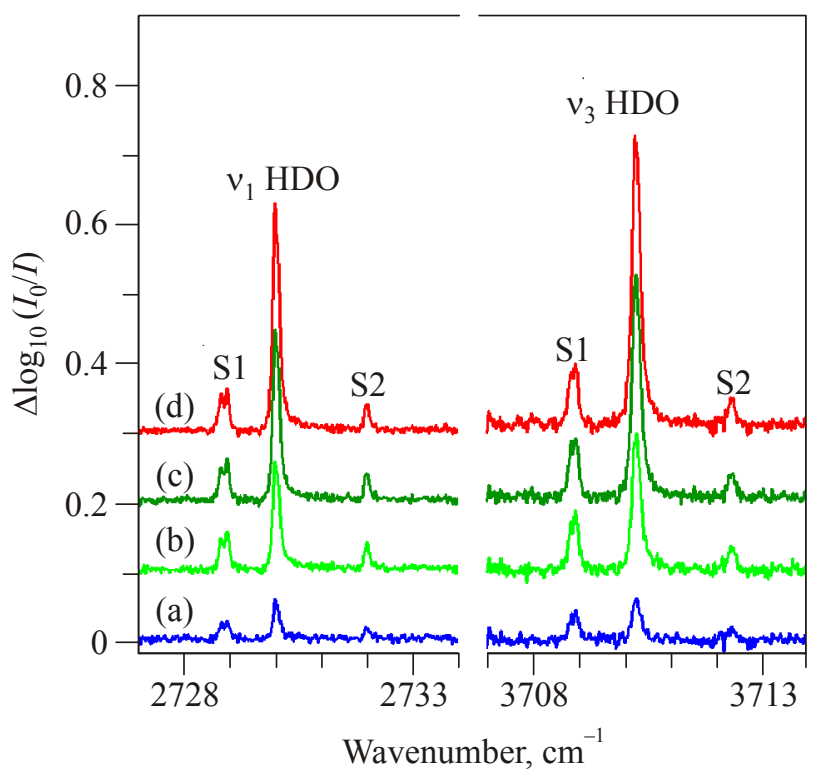

Fig. 2. (Color online) The same series of IR difference spectra shown in Fig. 1 showing the $v_{1}$ and $v_{3}$ HDO $a$-type rovibrational peaks. Note that both HDO rovibrational transitions show S1 and S2 satellite peaks. 
tector used in this study compared to the InSb detector used previously. However, we used the MCT detector specifically to record the $v_{2}$ bend rovibrational transitions shown in Fig. 1 since this mode has both relatively strong $a$-type and $b$-type rovibrational peaks. These $v_{2}$ HDO absorptions cannot be recorded with the InSb detector because the peaks are below the band gap for that detector. Further, the two $v_{2}$ HDO transitions shown in Fig. 1 are well separated and do not overlap with any other HDO, $\mathrm{D}_{2} \mathrm{O}$, or $\mathrm{H}_{2} \mathrm{O}$ peaks allowing us to measure the integrated intensity of both of these features. As we will show in this paper, this makes the $v_{2}$ HDO fundamental an excellent spectroscopic probe to determine the reason for the apparent lack of $b$-type satellite peaks. Another advantage of HDO compared to $\mathrm{H}_{2} \mathrm{O}$ or $\mathrm{D}_{2} \mathrm{O}$ is that HDO does not have complications in the intensity analysis due to different nuclear spin states. The in situ photolysis of DCOOD in solid $p \mathrm{H}_{2}$ also produces growth in the intensities of $\mathrm{D}_{2} \mathrm{O}$ and $\mathrm{H}_{2} \mathrm{O}$ rovibrational peaks as well [1]. However, for $\mathrm{H}_{2} \mathrm{O}$ and $\mathrm{D}_{2} \mathrm{O}$ these species are also produced in excited nuclear spin states which relax with time. As we will show, this complicates the intensity analysis of the $\mathrm{H}_{2} \mathrm{O}$ satellite peaks.

Shown in Fig. 3 are rovibrational transitions out of the lowest rotational state for the two vibrational modes of $\mathrm{D}_{2} \mathrm{O}$. In this case, neither the $b$-type $v_{2}$ bend nor the $a$-type $v_{3}$ asymmetric stretch shows evidence of satellite peaks. As we reported earlier [1], this is what we expect if the carriers of the satellite peaks are only formed when one of the photoproducts chemically reacts with the $\mathrm{pH}_{2}$ host. Starting from a DCOOD precursor molecule, the only way to generate $\mathrm{D}_{2} \mathrm{O}$ is via the molecular photochannel that produces $\mathrm{CO}+\mathrm{D}_{2} \mathrm{O}$. Indeed, gas phase measurements on $\mathrm{HCOOH}$ indicate the molecular photoproducts (i.e., $\mathrm{CO}+$

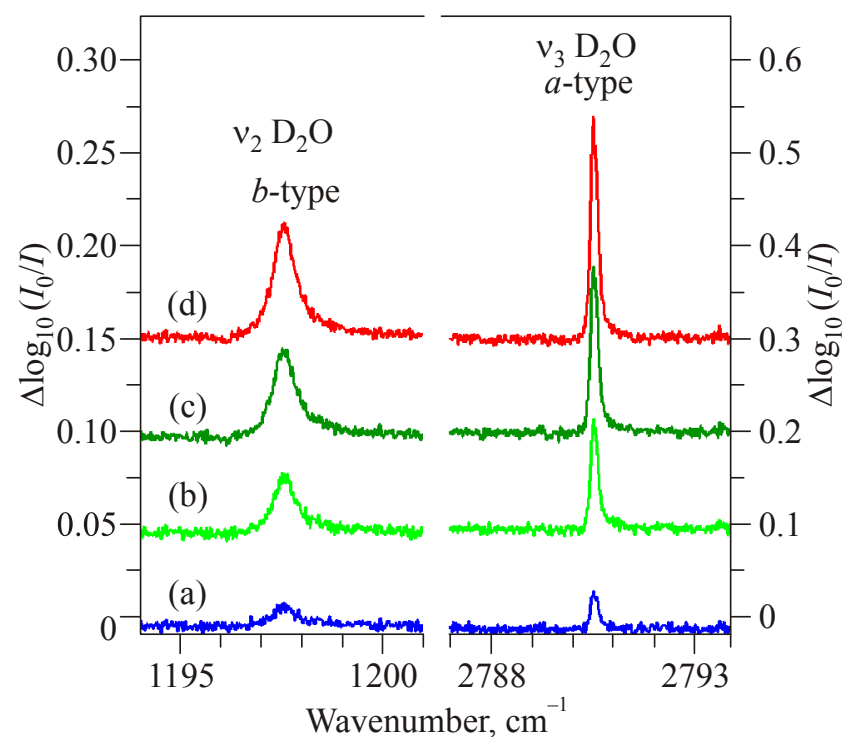

Fig. 3. (Color online) The same series of IR difference spectra shown in Fig. 1 now displaying the $b$-type $v_{2} \mathrm{D}_{2} \mathrm{O}$ and $a$-type $v_{3}$ $\mathrm{D}_{2} \mathrm{O}$ rovibrational peaks. Note that neither of the $\mathrm{D}_{2} \mathrm{O}$ rovibrational peaks show evidence of $\mathrm{S} 1$ and $\mathrm{S} 2$ satellite peaks.
$+\mathrm{H}_{2} \mathrm{O}$ and $\mathrm{CO}_{2}+\mathrm{H}_{2}$ ) are important at $193 \mathrm{~nm}$ and strongly favor production of $\mathrm{CO}+\mathrm{H}_{2} \mathrm{O}$ channel [2,3]. In fact, photochemical studies of $\mathrm{HCOOH}$ in $\mathrm{Ar}$ and $\mathrm{Xe}$ matrices show that only the molecular photochannels are observed in these condensed phase studies [26]. While some isotopic scrambling does occur to the DCOOD precursor molecule prior to photolysis, which can complicate the determination of the mechanistic origins of the HDO or $\mathrm{H}_{2} \mathrm{O}$ photoproducts, the $\mathrm{D}_{2} \mathrm{O}$ must come directly from the DCOOD precursor. This is consistent with the absence of any satellite peaks detected for the $\mathrm{D}_{2} \mathrm{O}$ molecule because there is no way to form $\mathrm{D}_{2} \mathrm{O}$ from reactions of the OD photofragment with the $p \mathrm{H}_{2}$ host. Thus, while not observing satellite peaks for the $b$-type $v_{2}$ transition is inconclusive, the lack of satellite peaks for the $a$-type transition strongly supports our assignment of why the satellite peaks form. For example, in other experiments using our InSb detector and thus with higher $\mathrm{S} / \mathrm{N}$ in the $v_{3}$ region, we have not observed any hint of $a$-type $\mathrm{D}_{2} \mathrm{O}$ satellite peaks.

Finally for completeness, we show in Fig. 4 the analogous rovibrational peaks for $\mathrm{H}_{2} \mathrm{O}$. Once again, we only observe satellite peaks centered around the $a$-type rovibrational transition and not for the $b$-type peak of the $v_{2}$ bend of $\mathrm{H}_{2} \mathrm{O}$. Tracking the mechanistic origins of the $\mathrm{H}_{2} \mathrm{O}$ satellite peaks is less clear than for $\mathrm{D}_{2} \mathrm{O}$ since some of the signal could result from the photochemistry of minor isotopomers present in the $\mathrm{pH}_{2}$ solid. Further, it seems that some of the $\mathrm{H}_{2} \mathrm{O}$ satellite peak intensity comes from DCOOD via photoproduction of OD followed by exchange reactions with the $\mathrm{pH}_{2}$ host or secondary photolysis that ultimately produces $\mathrm{OH}$. Thus, while it is difficult to tease out contributions from photolysis of DCOOH from DCOOD, the data indicates that some of the $\mathrm{H}_{2} \mathrm{O}$ satellite peaks are

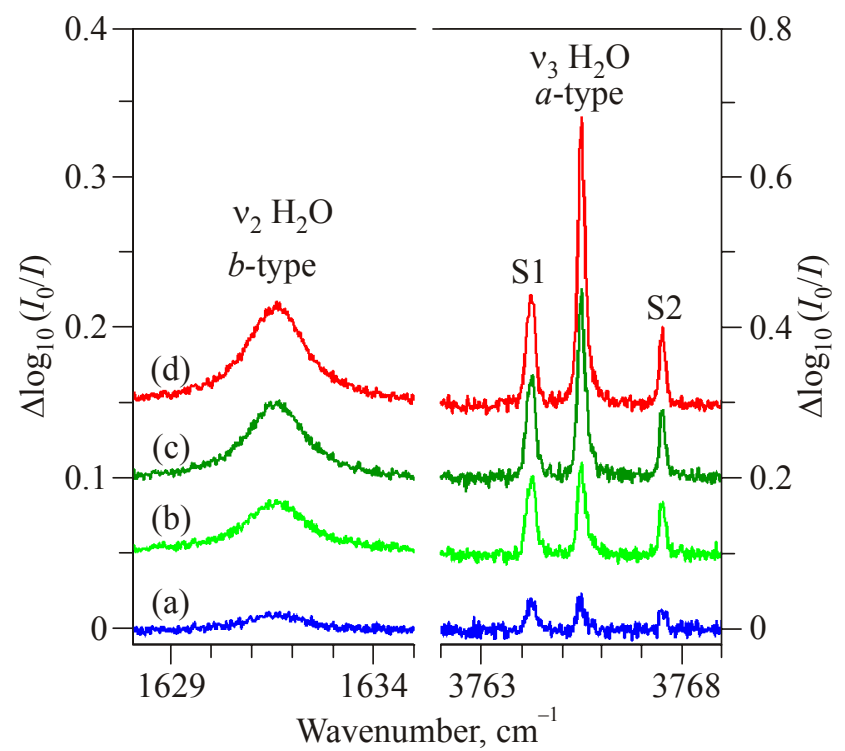

Fig. 4. (Color online) The same series of IR difference spectra shown in Fig. 1 now displaying the $b$-type $v_{2} \mathrm{H}_{2} \mathrm{O}$ and $a$-type $v_{3}$ $\mathrm{H}_{2} \mathrm{O}$ rovibrational peaks. Note that only the $a$-type rovibrational peak shows clear S1 and S2 satellite peaks. 
produced from photolysis of DCOOD. In the case of DCOOD photolysis only the $\mathrm{O}$ atom comes from the precursor, and all the $\mathrm{H}$ atoms in the cluster come from reactions with the $p \mathrm{H}_{2}$ solid.

An important clue as to why the $b$-type satellite peaks are not detected is provided by careful intensity analysis of the $v_{2}$ HDO rovibrational peaks. The satellite peaks rely on the transition strength of the $\mathrm{HDO}\left(\mathrm{H}_{2} \mathrm{O}\right)$ rovibrational transition to be detected, and therefore satellite peaks should be evident for all the rovibrational transitions of HDO $\left(\mathrm{H}_{2} \mathrm{O}\right)$, not just the $a$-type transitions. Shown in Fig. 5, $a$ are plots of the integrated intensity of various absorption features versus time for the experiment depicted in the earlier spectral figures. The grey vertical bar in Fig. 5, $a$ indicates the time and duration of the $193 \mathrm{~nm}$ photolysis exposure for this experiment. As can be seen in Fig. 5, $a$, before photolysis the intensity of the HDO monomer $b$-type and $a$-type rovibrational transitions are both quite small simply due to the low concentration of HDO prior to photolysis. During photolysis, the intensity of the
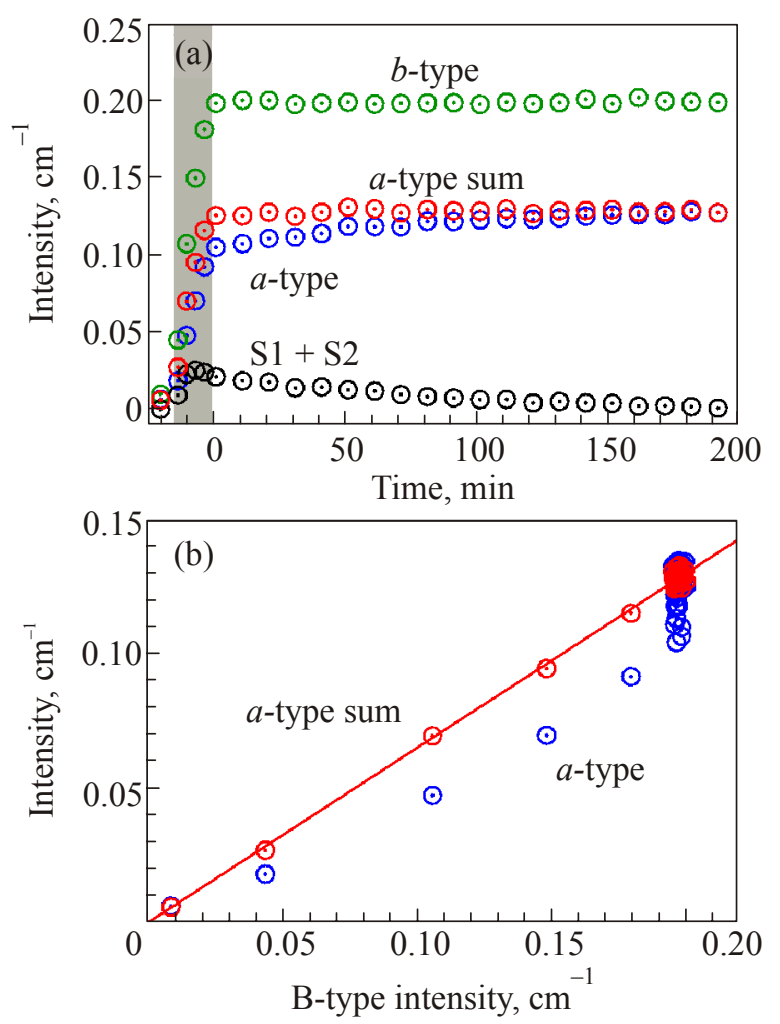

Fig. 5. (Color online) (a) A plot of the integrated intensities of the $v_{2}$ HDO $b$-type, $a$-type, S1 $+\mathrm{S} 2$, and sum of the $a$-type and S1 + $\mathrm{S} 2$ satellite peaks ( $a$-type sum) versus time. The grey shaded area represents the timing of the $193 \mathrm{~nm}$ photolysis during the experiment. Note the temporal profile of the $a$-type sum and $b$-type is very similar. (b) A correlation plot (blue circles) of the integrated intensity of the $a$-type transition versus the $b$-type transition generated from the data shown in (a). The corresponding correlation plot (red circles) for $a$-type sum versus $b$-type displays a strong linear correlation with a fitted slope of 0.6492(14) determined from a least squares fit of the data to $y=a x$ (solid red line).
HDO monomer transitions both increase as well as the S1 and S2 satellite peaks. The plot of the $b$-type transition intensity rapidly increases during photolysis, and then remains constant after photolysis. This is the expected behavior for the photolytic production of HDO if no intermediate species are detected. However, for the $a$-type transition the intensity is observed to continue to increase slightly after photolysis is complete (see Fig. 5,a). This is due to the mechanism by which the HDO monomer is produced and because we resolve the $a$-type satellite peaks; we can monitor the intensity or concentration of these two species separately. The HDO photoproduct cannot be generated directly from the DCOOD precursor, instead some of the photoexcited DCOOD molecules dissociate along the DCO + OD radical photochannel. The nascent OD either reacts directly with the $p \mathrm{H}_{2}$ host, or as we suspect, becomes thermally equilibrated and then reacts with the $p \mathrm{H}_{2}$ host via a tunneling reaction mechanism that produces HDO trapped next to an $\mathrm{H}$ atom or a vacancy. The $\mathrm{OH}+\mathrm{H}_{2} \rightarrow \mathrm{H}_{2} \mathrm{O}+\mathrm{H}$ hydrogen abstraction reaction is well characterized and is exothermic $(-7450 \mathrm{~K})$ and has a low barrier $(3060 \mathrm{~K})[27,28]$. The presence of the $\mathrm{H}$ atom or vacancy next to the HDO molecule slightly perturbs the $1_{01} \leftarrow 0_{00} a$-type rovibrational transition such that it lifts the three-fold upper state $M_{J}$ degeneracy and splits the transition into two peaks (e.g., S1 and S2). Once the satellite peaks are produced via this mechanism, the intensity starts to decrease as the HDO molecules in this metastable solvation site decay back to a well isolated HDO molecule in the ground rotational state. Due to the low temperature, relaxation of the quantum solid around the newly formed HDO molecule by either mechanism (movement of the $\mathrm{H}$ atom or vacancy away from the HDO molecule) would be expected to occur with such a slow time constant (e.g., 121(7) min). Consistent with this assignment, the sum of the S1 and S2 satellite peaks intensities decay with time after photolysis while the $a$-type HDO monomer transition increases. Furthermore, the decrease in the sum of the two satellite peak integrated intensities matches quantitatively the increase in the intensity of the $a$-type HDO monomer transition. Thus, if the sum of the satellite intensities $(\mathrm{S} 1+\mathrm{S} 2)$ is added to the intensity of the $a$-type HDO monomer transition, the resulting intensity profile ( $a$-type sum) matches qualitatively the $b$-type where the contributions between the satellite and monomer peaks cannot be separately measured using FTIR spectroscopy. This implies that the broad $b$-type absorption profile includes contributions from the satellite peaks, but these contributions are just not spectroscopically resolved.

To further show that $b$-type satellite peaks are also present in the spectra presented in Fig. 1, but just not spectroscopically resolved, we show in Fig. 5, $b$ integrated intensity correlation plots. If we make a plot of the $a$-type intensity versus the $b$-type intensity (blue circles), we observe a nonlinear correlation. This indicates that the po- 
pulation or concentration of the species measured by $a$ type transition does not match exactly the population probed by the $b$-type transition. More specifically, after photolysis the $a$-type HDO monomer transition continues to increase in intensity while the $b$-type HDO intensity remains constant (vertical scatter of data at the high $b$-type intensity). This simply shows that the $b$-type transition reflects the total of satellite and monomer peaks, while for the $a$-type transition these two contributions are spectroscopically resolved and can be detected separately. If instead we make a correlation plot of the $a$-type sum (monomer plus satellites) versus the $b$-type transition intensity, then we observe a strong linear correlation with a fitted slope of 0.6489(17). Indeed, this is what is expected based on the transitions strengths of $a$-type and $b$-type HDO $v_{2}$ bend transitions, which show that the transition dipole has a larger projection along the inertial $B$ axis [29]. This also shows that the approximation that the transition strength is the same for the monomer and satellite peaks is valid. Thus, the satellite peaks are present for the $b$-type transitions, but just not spectrally resolved.

To further test this explanation, we looked at the details of the $b$-type lineshape right after photolysis is complete, when the satellite peaks have the greatest intensity, and after the sample has been allowed to fully equilibrate and the satellite peaks have decayed to zero. Shown in Fig. 6 are expanded views of the $b$-type transitions of $\mathrm{D}_{2} \mathrm{O}$ (a)

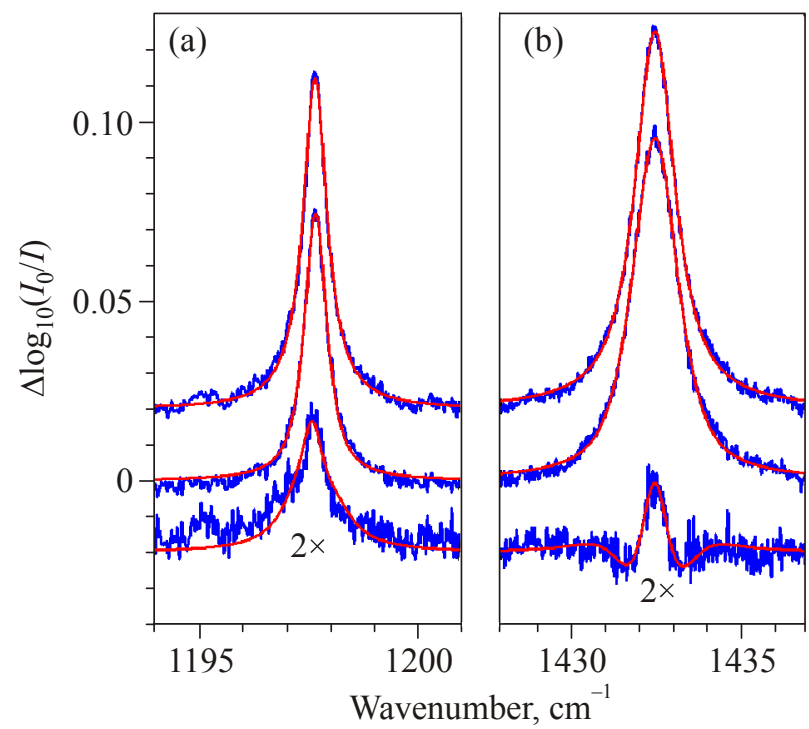

Fig. 6. (Color online) (a) Expanded view of the absorption spectra (blue) of the $b$-type $v_{2} \mathrm{D}_{2} \mathrm{O}$ and (b) $v_{2}$ HDO rovibrational transitions and least squares fits to a pseudo-Voigt lineshape (red). Spectra are offset vertically for ease of comparison. The top spectrum is recorded 496.5 min after the middle spectrum, which is recorded immediately after photolysis with a 3 min acquisition time. The bottom difference spectra (top - middle) is generated from these two spectra with the intensity multiplied by a factor of two $(2 \times)$. For $\mathrm{D}_{2} \mathrm{O}$ the peak intensity increases with time, but for HDO the difference lineshape is more complex due to contributions from satellite peaks (see text for details). and HDO (b) for the $v_{2}$ bend vibrational mode (blue trace). Each transition is then least-squares fit to a pseudo-Voigt lineshape [30] and the fitted lineshape is also shown in Fig. 6 (red trace), so that the difference lineshape can be examined at greater detail. For $\mathrm{D}_{2} \mathrm{O}$, as shown previously, there are no satellite peaks produced and the difference between the two lineshapes just shows overall growth in the intensity of this feature. This can easily be explained since some of the $\mathrm{D}_{2} \mathrm{O}$ molecules are produced in excited nuclear spin states during photolysis and thus right after photolysis is complete, the intensity of the $b$-type transition of $\mathrm{D}_{2} \mathrm{O}$ out of the lowest rotational level $\left(0_{00}\right)$ increases in intensity as the para- $\mathrm{D}_{2} \mathrm{O}$ molecules in excited rotational levels $\left(1_{01}\right)$ convert to the lower energy ortho- $\mathrm{D}_{2} \mathrm{O}$ spin state [1]. Indeed, we have shown in these earlier studies that the growth is first-order with a rate constant consistent with the literature value for the nuclear spin conversion rate constant of $\mathrm{D}_{2} \mathrm{O}[1,5]$. However, the difference spectrum generated for the analogous $b$-type HDO rovibrational transition shows a qualitatively different behavior. Instead in this case, the peak appears to narrow slightly after photolysis is complete. This is shown in Fig. $6, b$ by the difference spectrum that increases at the center line but has two dips near the wings. This behavior is consistent with there being two contributions to the observed lineshape whose relative concentrations change with time after the photolysis is complete. However, the total integrated intensity remains constant because the absorption strengths of both the satellite and monomer transitions are the same. Interestingly, the effect of these changes in relative concentrations is just at the level that can be detected using this spectroscopic data. This again is consistent with approximately $15 \%$ of the $b$-type signal being due to satellite peaks right after photolysis is complete, which then decays with time. It does not appear that the contributions of the satellite peaks can be extracted solely from the $b$-type rovibrational data, but fortunately the satellite peaks can easily be resolved using the narrower $a$-type rovibrational transition. In fact, using the gas phase absorption strength for the HDO $v_{2}$ bend $(56.91 \mathrm{~km} / \mathrm{mol})$ [31] appropriately scaled for the weaker $a$-type rovibrational transition, we estimate using Beer's law that concentration of HDO molecules in this metastable solvation state is $1.1 \mathrm{ppm}$ right after photolysis is complete under the conditions of this experiment.

\section{Summary}

In preliminary studies of the $193 \mathrm{~nm}$ in situ photolysis of DCOOD in solid $p \mathrm{H}_{2}$ at temperatures below $2 \mathrm{~K}$ we identified satellite peaks for the $a$-type rovibrational transitions of all three fundamental modes of HDO which are produced during photolysis and decay in intensity after photolysis is complete. We assigned these satellite features to $\mathrm{H} \cdots \mathrm{HDO}$ radical clusters that form by reactions of OD with the $p \mathrm{H}_{2}$ host. However, in these initial studies we were confused as to why we did not observe the analogous 
satellite transitions for $b$-type HDO rovibrational transitions. In the present paper, we use the $v_{2}$ bend of HDO to show that indeed the satellite peaks are present for $b$-type transitions, but are difficult to detect due to the greater fwhm of the $b$-type transitions of HDO in solid $p \mathrm{H}_{2}$. Plots of integrated intensity versus time after photolysis show that both the HDO monomer and satellite peaks contribute to the $b$-type $1_{11} \leftarrow 0_{00}$ rovibrational absorption intensity, while for the $a$-type transition the two contributions are spectrally resolved and can therefore be separated. The present study also shows that the absorption strength of the $a$-type HDO monomer and satellite peaks are equal, such that the gas phase absorption strength can be used to calculate the approximate concentration of HDO molecules in this metastable state.

The additional spectral data presented in this paper is consistent with the carriers of the satellite peaks being either H $\cdots$ HDO radical clusters or HDO next to a substitutional vacancy and represents a significant step forward in the FTIR spectroscopy of these satellite peaks. The IR spectroscopy shows that the satellite spectra (splitting and relative intensities) are very similar for all three vibrational modes of HDO and thus primarily reflect the lifting of the $(2 J+1)$ spatial degeneracy of the upper $1_{01}$ HDO rotational state. The ground rotational state $\left(0_{00}\right)$ is non-degenerate. However, a complete assignment of the satellite peaks is still lacking. Indeed it may prove difficult to distinguish between the two potential assignments presented here using just IR spectroscopy. We hope the preset experimental findings stimulate theoretical calculations of the two presented scenarios: (i) rotational motion of HDO next to an $\mathrm{H}$ atom in solid $p \mathrm{H}_{2}$ and (ii) rotation of $\mathrm{HDO}$ next to a vacancy in solid $p \mathrm{H}_{2}$. In either case the identification of these HDO satellite peaks in UV irradiated DCOOD doped $p \mathrm{H}_{2}$ solids offer new potential insights into the details of radiation effects in chemically doped quantum solids. We are currently using the spectroscopy developed here to study the details of the photolysis of DCOOD in solid $p \mathrm{H}_{2}$ aimed at correlating the decay in the satellite peaks with the growth of other species within the solid.

\section{Acknowledgments}

W.R.W. was supported partially by the Research Experiences for Undergraduates (REU) Program of the National Science Foundation under Award Number CHE 08-51931. The authors thank the National Science Foundation for its generous support through Grant CHE 08-48330.

1. K.A. Kufeld, W.R. Wonderly, L.O. Paulson, S.C. Kettwich, and D.T. Anderson, J. Phys. Chem. Lett. 3, 342 (2012).

2. H. Su, Y. He, F. Kong, W. Fang, and R. Liu, J. Chem. Phys. 113, 1891 (2000).

3. E. Martinez-Nunez, S.A. Vazquez, I. Borges, Jr., A.B. Rocha, C.M. Estevez, J.F. Castillo, and F.J. Aoiz, J. Phys. Chem. A109, 2836 (2005).
4. P.F. Bernath, Spectra of Atoms and Molecules, Oxford University Press, Inc., Oxford (2005).

5. M.E. Fajardo, S. Tam, and M.E. DeRose, J. Mol. Struct. 695 , 111 (2004).

6. A.V. Ivliev, A.Y. Katunin, I.I. Lukashevich, V.V. Sklyarevskii, V.V. Suraev, N.I. Filippov, and V.A. Shevtsov, JETP Lett. 36, 472 (1982).

7. A.S. Iskovskhih, A.Y. Katunin, I.I. Lukashevich, V.V. Sklyarevskii, V.V. Suraev, N.I. Filippov, and V.A. Shevtsov, Sov. Phys. JETP 64, 1085 (1986).

8. T. Miyazaki, K.P. Lee, K. Fueki, and A. Takeuchi, J. Phys. Chem. 88, 4959 (1984).

9. T. Kumada, N. Kitagawa, T. Noda, J. Kumagai, Y. Aratono, and T. Miyazaki, Chem. Phys. Lett. 288, 755 (1998).

10. T. Miyazaki, S. Mori, T. Nagasaka, J. Kumagai, Y. Aratono, and T. Kumada, J. Phys. Chem. A104, 9403 (2000).

11. T. Kumada, S. Mori, T. Nagasaka, J. Kumagai, and T. Miyazaki, J. Low Temp. Phys. 122, 265 (2001).

12. T. Kumada, M. Sakakibara, T. Nagasaka, H. Fukuta, and J. Kumagai, J. Chem. Phys. 116, 1109 (2002).

13. T. Kumada, Phys. Rev. B68, 052301 (2003).

14. T. Kumada, Y. Shimizu, T. Ushida, and J. Kumagai, Radiat. Phys. Chem. 77, 1318 (2008).

15. M. Fushitani and T. Momose, Fiz. Nizk. Temp. 29, 985 (2003) [Low Temp. Phys. 29, 740 (2003)].

16. L. Andrews and X. Wang, J. Phys. Chem. A108, 3879 (2004).

17. L. Andrews and X. Wang, J. Chem. Phys. 121, 4724 (2004).

18. A.F. Andreev and I.M. Lifshitz, Sov. Phys. JETP 29, 1107 (1969).

19. D. Zhou, C.M. Edwards, and N.S. Sullivan, Phys. Rev. Lett. 62, 1528 (1989).

20. F. Operetto and F. Pederiva, Phys. Rev. B75, 064201 (2007).

21. L.O. Paulson and D.T. Anderson, J. Phys. Chem. A113, 1770 (2009).

22. M.E. Fajardo, in: Physics and Chemistry at Low Temperatures, L. Khriachtchev (ed.), Pan Stanford Publishing Pte. Ltd., Singapore (2011), p. 167.

23. I. Yokoyama, Y. Miwa, and K. Machida, J. Am. Chem. Soc. 113, 6458 (1991).

24. M.E. Fajardo and S. Tam, J. Chem. Phys. 115, 6807 (2001).

25. M.E. Fajardo and C.M. Lindsay, J. Chem. Phys. 128, 014505 (2008).

26. J. Lundell and M. Räsänen, J. Phys. Chem. 99, 14301 (1995).

27. M. Alagia, N. Balucani, P. Casavecchia, D. Stranges, G.G. Volpi, D.C. Clary, A. Kliesch, and H.-J. Werner, Chem. Phys. 207, 389 (1996).

28. M.D. Wheeler, D.T. Anderson, and M.I. Lester, Int. Rev. Phys. Chem. 19, 501 (2000).

29. S.A. Clough, Y. Beers, G.P. Klein, and L.S. Rothman, J. Chem. Phys. 59, 2254 (1973).

30. A.L. Stancik and E.B. Brauns, Vib. Spectrosc. 47, 66 (2008).

31. D.J. Swanton, G.B. Bacskay, and N.S. Hush, J. Chem. Phys. 84, 5715 (1986). 